Archives

$37 \mid 2006$

Quoi de neuf en Histoire Ancienne ? Le diable au Moyen Âge

\title{
Satan, Descartes et Kantorowicz
}

À propos de Satan hérétique

\section{Étienne Anheim}

\section{(2) OpenEdition \\ Journals}

\section{Édition électronique}

URL : http://journals.openedition.org/ccrh/3158

DOI : $10.4000 /$ ccrh.3158

ISSN : 1760-7906

\section{Éditeur}

Centre de recherches historiques - EHESS

Édition imprimée

Date de publication : 1 juin 2006

Pagination : 199-208

ISSN : 0990-9141

Référence électronique

Étienne Anheim, «Satan, Descartes et Kantorowicz », Les Cahiers du Centre de Recherches Historiques [En ligne], 37 | 2006, mis en ligne le 08 septembre 2011, consulté le 19 avril 2019. URL : http:// journals.openedition.org/ccrh/3158; DOI : 10.4000/ccrh.3158

Ce document a été généré automatiquement le 19 avril 2019.

Article L.111-1 du Code de la propriété intellectuelle. 


\title{
Satan, Descartes et Kantorowicz
}

\author{
À propos de Satan hérétique
}

\author{
Étienne Anheim
}

1 Comme dans beaucoup d'autres livres d'Alain Boureau, il règne dans Satan hérétique une atmosphère énigmatique, un air d'enquête policière; une telle impression de lecture ne peut qu'éveiller la curiosité du lecteur, peu habitué à passer sans transition du Moyen Âge à Conan Doyle, et le pousser à comprendre son origine ${ }^{1}$. Ce parfum d'étrangeté semble le fruit de principes de construction repris de livre en livre : le choix de sujets singuliers et apparemment ponctuels, tant dans la thématique que dans la chronologie ; la rapidité des considérations introductives présentant la démarche et les étapes successives de la démonstration; enfin, trait sans doute le plus frappant qui fait voisiner Alain Boureau avec les romanciers de la modernité, le goût de l'ellipse : le passage d'un chapitre à l'autre est souvent l'occasion d'une dramatisation de la narration qui ne déteste pas recourir à des césures, des blancs, des sauts chronologiques et thématiques.

2 En ce sens, le premier aspect notable de ce livre est sa composition, c'est-à-dire cette manière d'écrire « en pointillés » en quelque sorte, en sautant d'un dossier à l'autre tout en laissant au lecteur le soin de reconstruire certains enchaînements, comme une façon de ne pas surdéterminer le discours de l'historien et d'ouvrir le texte à l'interprétation. En songeant à ces choix narratifs, une phrase vient alors à l'esprit :

On peut distinguer deux types d'historien : l'archéologue qui découvre de nouvelles sources sur le passé et le narrateur qui combine autrement les sources disponibles. Le narrateur s'installe en un point du passé où tout est déjà là : les événements ont eu lieu, le commentaire, surabondant, a déjà été écrit, modifié, nuancé. Le narrateur s'assoit, cependant, et gourmand de surprise, déclare : ce n'est point ainsi que les choses se sont passées. Je vais vous raconter².

3 C'est ainsi qu'Alain Boureau lui-même qualifiait le travail d'historien d'Ernst Kantorowicz dans son essai biographique publié en 1990. À propos de l'historien des Deux corps du roi, il a encore d'autres formules aux consonances familières :

L'historien privilégie l'étonnement devant le détail étrange comme méthode de découverte historique $»^{3}$; [ou encore] Il ne fut jamais un spécialiste ni le gardien d'un champ historique. Ou plutôt, il fut un nomade de la spécialisation : arrêté par 
quelque objet dont il pressentait la bizarrerie, il s'en constituait le spécialiste instantané et provisoire ${ }^{4}$.

4 Ainsi, en relisant ce livre consacré à Kantorowicz, on a l'impression de trouver l'exposé méthodologique qui faisait un peu défaut dans Satan Hérétique. Dans l'interprétation de Kantorowicz, deux observations paraissent en particulier pouvoir être retournées « contre » le biographe. D'une part, Alain Boureau écrit :

[...] à lire l'énoncé particulièrement restreint d'une étude, on sait d'avance que quelque fulgurance naîtra de l'infime pointe du silex ${ }^{5}$.

D'autre part, il juge à propos des Deux corps du roi :

Le gain consiste à se doter d'un lien causal entre les réalités socio-économiques certaines qui font naître (ou renaître) l'État au $\mathrm{XV}^{\mathrm{e}}$ siècle et les mentalités, qui ne peuvent recevoir directement la nécessité étatique. L'économie substantielle porte sur la croyance. L'analyse de Kantorowicz nous permet de comprendre qu'entre l'action et la pensée, entre la pratique et la croyance, le langage glisse son efficacité propre, dans l'éclat joyeux des surfaces ${ }^{6}$,

idée qu'Alain Boureau ramasse quelques pages plus loin dans une formule qui rappelle Michel Foucault : « l'énoncé ne décrit pas, il agit »?

7 En somme, il s'agirait de travailler à partir d'un objet très circonscrit, « l'infime pointe du silex » - par exemple la question des démons dans les sources savantes des décennies entourant le tournant des XIII et XIVe siècle - pour reconstituer l'efficace d'un langage nouveau, celui d'une incrimination hérétique des individus soupçonnés de commerce avec le diable, cette reconstruction débouchant sur une interprétation plus large, qui proposerait de nouveaux liens de causalité entre réalités socio-économiques, constructions politiques et mentalités. Voilà bien, à travers le commentaire du travail de Kantorowicz, l'exposé de la méthode et de l'objectif visé par Satan hérétique.

\section{La question de l'histoire intellectuelle}

8 Le pivot de la démonstration est constitué par l'étude des sources scolastiques, c'est-àdire de textes très techniques, dans lesquels des philosophes, des juristes mais surtout des théologiens redéfinissent peu à peu les rapports entre le crime d'hérésie (qui, à l'origine, désigne une opinion fausse) et les créatures diaboliques et démoniaques, jusqu'au point où apparaît une nouvelle signification du terme : l'hérésie devient un fait et non plus une opinion, et, au premier chef, le fait d'avoir pactisé avec le diable et les démons d'une manière ou d'une autre. Le livre reconstitue la genèse intellectuelle de cette conception à partir d'une série de notions - fait, sacrement, pacte, volonté du sujet, possession - qui se recombinent entre les mains des théologiens pour produire cette nouvelle synthèse, ouvrant la voie à la chasse aux sorcières à partir du xve siècle. La thèse du livre est donc que cette mutation intellectuelle serait à l'origine d'un phénomène sociopolitique, affirmation qui est également une prise de position épistémologique soulignant l'importance des représentations, y compris abstraites et savantes, dans la structuration historique des sociétés.

L'idée proposée est précisément celle d'une efficacité de l'énoncé : la production d'un énoncé tel que « le pacte avec le diable existe bien, et il est hérétique », finit par avoir une action concrète sur la société occidentale, dont il modifie non seulement les représentations, mais aussi les pratiques. C'est cette idée de méthode qui sous-tend la 
démarche d'Alain Boureau: étudier la constitution d'énoncés efficaces, socialement performatifs en quelque sorte.

Dans cette mesure, le recours aux sources savantes et à l'histoire des doctrines s'explique très bien: elles sont le lieu privilégié de production de ces énoncés. Ce projet est, discrètement, la revendication d'une certaine manière de faire de l'histoire dont il faut souligner deux aspects qui méritent discussion, "un gain et une économie», comme dirait Alain Boureau à propos de Kantorowicz. Le gain est de trouver une base ferme aux assertions de l'historien. À ce titre, il paraît à nouveau nécessaire d'opérer un retour dans le passé, en 1989, avec l'article sur "l'histoire restreinte des mentalités », en réponse à l'éditorial des Annales sur le tournant critique ${ }^{8}$. Dans cet article, Alain Boureau critiquait la dérive de l'histoire des mentalités : « la problématique s'est transformée partiellement en discipline positive " ${ }^{9}$. En d'autres termes, la question des «mentalités » est devenue un objet historique, et non plus une méthode pour une histoire qui se rêverait «totale». Mais en faisant référence à Kantorowicz et à Foucault ${ }^{10}$, Alain Boureau tente en 1989 de définir ce qu'il appelle « une autre voie, plus rigoureuse $»^{11}$, en développant un exemple, emprunté, et ce n'est pas un hasard, au champ de l'histoire des doctrines médiévales, en l'occurrence l'usage d'un adage de droit romain, quod omnes tangit, dans le contexte de la fin du Moyen Âge ${ }^{12}$. La ligne est clairement définie dans la conclusion :

Il s'agirait de trier le faisceau des relations qui lient les globalités aux agents historiques et de désigner une zone frontalière d'intrication où le même énoncé appartient simultanément et intégralement à un discours social déterminé et à des énonciations singulières. L'histoire restreinte des mentalités étudierait en somme l'incorporation du réel, en ses moments rares et structurants ${ }^{13}$.

Il semble, à relire ces passages, que Satan hérétique, de même que tout le travail historique consacré à l'anthropologie scolastique, est justement la concrétisation exacte de ce projet, et que pour Alain Boureau, la période 1280-1330 fut un de ces "moments rares et structurants». Contre une lecture mettant l'accent sur la rupture avec l'histoire des mentalités dont le texte de 1989 serait l'oraison funèbre, au profit de la construction d'un nouveau projet, une histoire intellectuelle rénovée ${ }^{14}$, il me semble voir ici plutôt, historiographiquement, une continuité de l'un à l'autre ${ }^{15}$. L'histoire intellectuelle selon Alain Boureau est justement le terrain de cette histoire restreinte des mentalités : en ce sens, cette histoire intellectuelle ne viserait pas tant un objet qu'une méthode; elle garderait l'ambition d'une explication générale qui se rattacherait directement à l'histoire-problème et à l'histoire des mentalités selon Marc Bloch ${ }^{16}$. Le gain est donc d'avoir implicitement reformulé sur de nouvelles bases méthodologiques le vieux programme d'histoire des mentalités et de psychologie historique des années vingt, en lui donnant une rigueur nouvelle et des outils inédits.

L'économie réside pour sa part dans cette "efficacité " prêtée aux énoncés. Plutôt que d'essayer de saisir des "mentalités vagues », il s'agit de retrouver les formes de discours qui les structurent. C'est toutefois également là que peut s'ouvrir la discussion avec la méthode du livre. Si la démonstration sur la structuration du discours démonologique au début du XIVe siècle et ses effets sur le XVe siècle est frappante, elle nécessite toutefois que le lecteur suive l'auteur dans sa confiance en l'efficacité des énoncés, alors que la démarche suivie tend à évacuer la question de la socialisation de ces représentations savantes. Pourtant le contexte social parait contraindre doublement l'énoncé. En amont, il contraint ses conditions de production: le livre laisse apercevoir que la structure d'opposition entre franciscains et dominicains est un puissant moteur de construction des énoncés, sur lequel l'auteur passe rapidement. Puis, en aval, il s'arrête au moment où le 
discours démonologique s'est infléchi, autour de 1330, sans que l'on voieles formes d'acculturation dans la seconde moitié du XIVe siècle, par lesquelles l'énoncé agit sur le social $^{17}$.

\section{Satan hérétique et l'historiographie médiévale}

13 On est tenté de lire dans cette démarche l'affirmation répétée d'une prédilection de l'école médiévistique française pour l'anthropologie, y compris sous sa forme intellectuelle, par rapport à la sociologie. Ces choix poussent à chercher derrière Satan hérétique non seulement une méthode, mais des thèses d' " histoire globale ".

Concernant le Moyen Âge d'abord, deux propositions paraissent particulièrement importantes. La première concerne les sources scolastiques et doctrinales de la fin du Moyen Âge. En effet, la méthode d'Alain Boureau ne consiste pas seulement à trouver dans ce type de source un support à une histoire des mentalités renouvelée, elle a aussi un effet sur le statut de ces sources elles-mêmes. Les historiens comme les philosophes se sont souvent plaints de l'isolement dans lequel étaient tenus ces milliers de pages de textes théologiques et philosophiques universitaires des XIII et XIVe siècle, qui étaient coupés à la fois d'une interprétation historique et philosophique ${ }^{18}$. Satan hérétique propose une manière de réintégrer la scolastique dans le matériau utilisable par l'historien, et en même temps, de lui donner sa véritable portée historique, c'est-à-dire d'avoir été le laboratoire des représentations de l'Occident de la fin du Moyen Âge, aussi bien pour ce qui concerne la conception de l'individu et du sujet que de la communauté, de la politique ou des échanges. À travers le livre se dessine l'idée d'une anthropologie générale des sociétés occidentales de la fin du Moyen Âge à partir de ces savoirs théologicophilosophiques.

Le corollaire de cette idée, seconde proposition à portée générale, est la continuité entre les derniers siècles du Moyen Âge et l'époque moderne. Le livre montre la genèse chez les clercs médiévaux d'une structure d'incrimination et de lecture du monde à travers l'hérésie et le pacte diabolique, qui connaît l'essentiel de son succès entre le XVe et le XVII siècle. La chronologie que retrace Satan hérétique est celle d'une rupture forte au XIII siècle, avec l'essor de nouvelles structures comme l'université, de nouvelles pratiques sociales comme le pacte et de nouveaux systèmes de représentations savantes. Ensuite, on peut considérer, si l'on suit la démonstration, que l'unité l'emporte jusqu'au XVII siècle : on aurait ainsi une nouvelle entité, constituée de la période XIII-XVII siècle, qui parait, du point de vue de l'histoire politique, très pertinente, même s'il ne faut pas oublier que toute périodisation est un choix qui comporte avantages et inconvénients. Cette approche pose toutefois une question, si on se rappelle évidemment la formulation féconde de Jacques Le Goff sur le « long Moyen Âge », désignant quasiment l'ensemble de la période

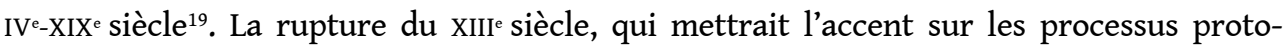
étatiques, économiques, culturels, paraît nuancer cette vision « continuiste ».

\section{Perspectives modernes}

16 Afin d'illustrer l'intérêt de cette prolongation dans l'époque moderne des mécanismes intellectuels mis en place entre les XIII et XIVe siècles, on peut, pour finir, revenir sur 
l'apport le plus important du livre, l'interprétation de la modernité politique et religieuse à travers la redéfinition de la personne humaine.

Par l'étude de la démonologie et de son entrée dans l'espace de répression politicoreligieux, Satan hérétique défend une thèse beaucoup plus générale, inscrite dans la chronologie XIII'-XVII ${ }^{e}$ siècle, à propos de la genèse des institutions modernes occidentales, églises (au pluriel) et états. En effet, les résultats du grand programme des années quatrevingt sur la genèse de l'État moderne, voient s'opposer deux tendances interprétatives, d'une part celles mettant l'accent sur la force de contrainte des constructions institutionnelles et juridiques permettant l'essor des nouvelles puissances étatiques, d'autre part celles privilégiant une lecture «contractualiste» du rapport entre souveraineté étatique et populations, fondé sur le dialogue politique. Or Alain Boureau déplace la réflexion en amont de cette opposition. Sa thèse tend à affirmer que les mécanismes d'émergence des formes étatiques de la fin du Moyen Âge, tant par le dialogue que par la puissance souveraine, de même que les formes de confessionnalisation religieuse, ont été rendues possibles par la construction intellectuelle et sociale d'une nouvelle configuration imaginaire ${ }^{20}$. Il s'agit d'un nouveau statut de la personne humaine, qui permet la représentation d'une société d'individus liés principalement par des formes contractuelles, individus dont la caractéristique anthropologique est d'être à la fois doté d'une capacité de volonté et de responsabilité qui les rend à même d'être contractant, et d'une fragilité, d'une potentialité d'aliénation qui les rend infiniment faillibles, et donc le cas échéant coupables.

Le pacte diabolique et hérétique serait un cas limite de cette nouvelle représentation sociale, par lequel se révélerait à la fois la puissance du sujet (capable de contracter un pacte avec le diable) et sa faiblesse (puisque c'est un geste absolument mauvais, une aliénation complète). Il y aurait dans cette mutation anthropologique l'un des points d'ancrage majeurs de la modernité, dont la thématique diabolico-hérétique ne serait qu'une sorte de catalyseur, de révélateur, et comme un effet secondaire.

Mais cet effet secondaire ne serait pas complètement accidentel : il y aurait comme une nécessité dans cette construction fantasmatique issue d'un moment de rationalisation intellectuelle. Les figures du diable et du sujet pleinement aliéné, complètement exclu de son humanité par sa faiblesse constitutive, et donc complètement éliminable par une société qui doit se défendre, représentent une manière radicale de penser l'altérité. Dans ce processus par lequel la société occidentale se réordonne à la fin du Moyen Âge et à l'époque moderne, il n'est pas sans importance que soit créé d'un même mouvement ce reste placé hors de l'humain, sous l'emprise diabolique. Il est comme la trace négative de l'évolution en cours: la construction progressive d'un Occident appuyé sur la raison et son universalisation passe par le rejet de cet autre diabolique, mais dans le même mouvement, la théorie du sujet faible place potentiellement l'altérité au cœur même de chacun. Le procès de civilisation et la raison scolastique enfantent ainsi la chasse aux sorcières sans l'ombre d'un paradoxe: elle ne font qu'exprimer la part de barbarie qu'elles voulaient rejeter, en la portant au contraire à un stade supérieur, c'est-à-dire en l'intériorisant complètement.

20 La mise au jour de cette structure de partage entre la raison et son autre, qu'on voudrait expulser du corps social sous la figure de l'hérétique diabolique, mais qui passe en réalité à l'intérieur de chaque sujet et qui peut se révéler aux yeux de la communauté par un simple factum, un fait, qui sera révélateur d'un autre fait, dissimulé, mais terrible, le pacte 
diabolique, est un élément fondamental pour la relecture de la modernité occidentale. On ne prendra qu'un exemple, celui d'un texte célèbre relu à la lumière de Satan hérétique :

Je supposerai donc, non pas que Dieu, qui est très bon et qui est la souveraine source de vérité, mais qu'un certain mauvais génie, non moins rusé et trompeur que puissant, a employé toute son industrie à me tromper; je penserai que le ciel, l'air, la terre, les couleurs, les figures, les sons, et toutes les autres choses extérieures, ne sont rien que des illusions et rêveries dont il s'est servi pour tendre des pièges à ma crédulité [...]; je demeurerai obstinément attaché à cette pensée ; et si, par ce moyen, il n'est pas en mon pouvoir de parvenir à la connaissance d'aucune vérité, à tout le moins il est en ma puissance de suspendre mon jugement. C'est pourquoi je prendrai garde soigneusement de ne recevoir en ma croyance aucune fausseté, et préparerai si bien mon esprit à toutes les ruses de ce grand trompeur, que, pour puissant et rusé qu'il soit, il ne me pourra jamais rien imposer ${ }^{21}$.

Ce passage de la première des Méditations métaphysiques de Descartes a donné lieu à une célèbre controverse entre Michel Foucault et Jacques Derrida ${ }^{22}$. Foucault soutenait que c'était l'acte fondateur du rejet de la folie par la raison hors de l'ordre du discours occidental $^{23}$; Derrida, au contraire, estimait qu'il n'y avait pas de partage, et que Descartes montrait que le cogito se développait sur la toile de fond de la folie toujours possible ${ }^{24}$. Mais il est intéressant de remarquer que ces deux philosophes nourris d'une formation classique ont tous deux interprétés la figure du mauvais génie comme l'image de la folie, même si Derrida prend quelques précautions sur ce point ${ }^{25}$. Or la lecture du livre d'Alain Boureau pourrait suggérer une nouvelle forme d'historicisation de ce texte en trouvant également dans cette figure une transposition du diable scolastique de la fin du Moyen Âge, d'autant que la dette de Descartes à l'égard de la scolastique médiévale est très importante.

Dans cette perspective, le moment fondateur de la philosophie du sujet chez Descartes apparaîtrait sous un jour un peu différent, celui d'une réflexion sur le sujet, sa volonté et sa faiblesse face au diable, dans la poursuite des termes posés par la scolastique des XIII et XIVe siècles. Il semble donc que la matrice intellectuelle qui produit les réflexions de Descartes ne soit pas seulement l'opposition baroque mise en scène par Foucault et acceptée par Derrida autour du couple folie/raison, mais aussi la pensée d'une altérité/ altération du sujet par une possible possession diabolique, et une méditation sur les moyens d'y échapper, ce qui en dernier lieu ramène à Dieu ${ }^{26}$. La proximité entre les Méditations, écrites en 1641, et l'affaire des possédés de Loudun en 1632, étudiée par Michel de Certeau ${ }^{27}$, renforce cette évidence : dans la perspective d'Alain Boureau, l'âge classique apparaît moins comme le moment inaugural d'un nouveau sujet occidental que comme une étape dans le déploiement d'une structure, celle du rapport entre des institutions et un sujet à la fois infiniment responsable et infiniment fragile. En quelque sorte, le sujet de Descartes rêve de repousser le malin génie, sans parvenir à effacer sa macule originelle. "Faisons comme si le diable n'existait pas ", dit la raison occidentale moderne, rejetant ainsi la part d'altérité qui pourtant a présidé à sa fondation même et qui continue à la travailler de manière souterraine à travers toute la modernitée ${ }^{28}$. En ce sens, Satan hérétique propose une anthropologie historique qui rend à la raison moderne sa part diabolique, ce qui conduit à laisser une dernière fois la parole à Alain Boureau parlant de Kantorowicz, et écrivant peut-être une autobiographie inconsciente : « Le rêve de tout historien: faire apparaître un phénomène qui était demeuré inaperçu tout en laissant des traces observables par quiconque $»^{29}$. 


\section{NOTES}

1. Le rapprochement entre le travail de l'historien et celui de l'enquêteur est évident dans d'autres livres d'Alain Boureau, comme par exemple Théologie, science et censure au XIII' siècle. Le cas de Jean Peckham, Paris, Les Belles Lettres, 1999, dont on rappellera les premières lignes de l'introduction, p. 1: «Ce livre aurait pu s'intituler L'archevêque et les cadavres. De fait, on y trouvera un cadavre qui saigne devant un meurtrier, de mauvais coups, de la véhémence, des fureurs, de la haine, de funestes destins ») et de l'épilogue, p. 333 : «au terme de cette enquête, Jean Peckham ne fait pas grande figure ». La comparaison rappelle bien sûr la thèse de Carlo Ginzburg sur le "paradigme indiciaire ", "Traces. Racines d'un paradigme indiciaire ", Mythes, emblèmes, traces. Morphologie et histoire, Paris, Flammarion, 1989 (éd. orig. italienne 1986), p. 139-180. On peut aussi y voir une alternative au parallèle entre le juge et l'historien - à moins qu'il ne s'agisse du juge d'instruction, qui, en fait, conduit l'enquête, selon la métaphore proposée par Marc Bloch en 1914 dans son discours de distribution des prix au lycée d'Amiens : "Nous sommes des juges d'instruction chargés d'une vaste enquête sur le passé ». Sur cette formulation et ses interprétations, voir Olivier Dumoulin, Marc Bloch, Paris, Presses de Sciences Po, coll. Références Facettes, 2000, p. 277-288.

2. Alain Boureau, Histoires d'un historien. Kantorowicz, Paris, Gallimard, Coll. «L'un et l'autre », 1990 , p. 16.

3. Ibid., p. 90.

4. Ibid., p. 119.

5. Ibid., p. 120.

6. Ibid., p. 150.

7. Ibid., p. 154.

8. Alain Boureau, « Propositions pour une histoire restreinte des mentalités ", Annales ESC, nov.déc. 1989, n6, p. 1491-1504.

9. Ibid., p. 1493.

10. Ibid., voir en particulier p. 1498.

11. Ibid., p. 1499.

12. Ibid., p. 1499-1501.

13. Ibid., p. 1501.

14. En ce sens, cette interprétation diffère de celle proposée par François Dosse dans L'Empire du Sens. L'humanisation des sciences humaines, Paris, La Découverte, 1995, p. 255-267 («Les représentations collectives : la sortie de l'histoire des mentalités »), à laquelle font écho plusieurs passages de La marche des idées. Histoire des intellectuels - histoire intellectuelle, Paris, La Découverte, 2003.

15. Dans cette perspective, on pourrait inscrire dans le même travail de reformulation problématique du programme d'histoire des mentalités des Annales, visant à en conserver la dimension critique, analytique et sociologique tout en en évitant les écueils, les propositions de Roger Chartier sur les représentations, voir en particulier « Histoire intellectuelle et histoire des mentalités » et «Le monde comme représentation », Au bord de la falaise. L'histoire entre certitudes et inquiétudes, Paris, Albin Michel, 1998, p. 27-66 et 67-86, ainsi que les recherches de Jean-Claude Schmitt et de Jérôme Baschet sur les images, qui forment un autre terrain possible pour construire une méthodologie plus rigoureuse dans ce domaine : Jean-Claude Schmitt, Le corps des images. Essais sur la culture visuelle au Moyen Âge, Paris, Gallimard, (Le temps des images), 2002, et 
Jérôme Baschet, Le sein du Père. Abraham et la paternité dans l'Occident médiéval, Paris, Gallimard. (Le temps des images), 2000.

16. Voir en particulier Marc Bloch, Les rois thaumaturges, Paris, Gallimard, 1983 (1 $1^{\text {ère }}$ éd. 1924), Bibliothèque des Histoires.

17. Cette observation rejoint certaines remarques de Martine Ostorero dans son compte rendu de Satan hérétique, Médiévales, 48, printemps 2005, p. 165-168.

18. On citera pour mémoire les propos d'Alain de Libéra dans Penser au Moyen Âge, Paris, Le Seuil, 1990, p. 28 : «Qu'est-ce à dire, sinon que le Moyen Âge intellectuel attend toujours son Foucault - celui qui saura, du même geste, rapatrier le Moyen Âge dans l'histoire de la philosophie, réintégrer l'histoire de la philosophie dans la philosophie même et fédérer le tout en une étude de la pensée qui serait à la fois histoire réfléchissante et réflexion sur l'histoire ? Que cet "individu historique" existe ou non n'est pas un problème : il s'agit là d'un programme de recherche collective, non de messianisme professionnel ", ainsi que ceux d'Anita GuerreauJalabert, dans l'Histoire culturelle de la France, t. 1, Le Moyen Âge, Paris, Le Seuil, 1997, p. 175 : «Ce qu'il faudrait donc étudier, ce sont les tensions d'ordre idéologique et pratique qui traversent le champ de la pensée théologique, dont l'interprétation socio-historique reste encore à faire ».

19. Jacques Le Goff, "Pour un long Moyen Âge », Europe, $\mathrm{n}^{\circ}$ 654, octobre 1983, p. 19-24, repris dans L'imaginaire médiéval, Paris, Gallimard, 1985 (nouvelle édition en 1991), p. 7-13, par exemple p. 10 : «Des structures fondamentales persistent dans la société européenne du IV au XIX ${ }^{\mathrm{e}}$ siècle qui permettent de saisir la cohérence de ces quinze siècles ».

20. Au sens de Cornelius Castoriadis, L'Institution imaginaire de la société, Paris, Le Seuil, 1975.

21. René Descartes, Méditations métaphysiques, Paris, Classiques Larousse, 1951, p. 32.

22. Concernant les interprétations opposées des deux philosophes, voir Michel Foucault, Histoire de la folie à l'âge classique, Paris, Gallimard, coll. Tel, 1972, p. 67-70, et la réponse faite à Jacques Derrida en 1971, « Mon corps, ce papier, ce feu », publiée dans Paideia en septembre 1971 et repris en appendice de l'édition de l'Histoire de la folie de 1972 dans la Bibliothèque des Histoires; d'autre part, voir l'article de Jacques Derrida, « Cogito et histoire de la folie », paru en 1963 dans la Revue de Métaphysique et de Morale et repris en 1967 dans le recueil L'Écriture et la Différence, Paris, Le Seuil, 1967, p. 51-97. Pour une présentation rapide de cette controverse, voir Marc Goldschmit, Jacques Derrida, une introduction, Paris, La Découverte, 2003, «La pensée de la folie. La controverse avec Foucault sur les rapports de l'histoire et de la philosophie », p. 159-170, ainsi que François Dosse, Histoire du structuralisme, II. Le chant du cygne, 1967 à nos jours, Paris, La Découverte, 1992, p. 36-39.

23. Michel Foucault, Histoire de la folie, p. 70: «Le cheminement du doute cartésien semble témoigner qu'au XVII siècle le danger se trouve conjuré et que la folie est placée hors du domaine d'appartenance où le sujet détient ses droits à la vérité : ce domaine qui, pour la pensée classique, est la raison elle-même. Désormais la folie est exilée. Si l'homme peut toujours être fou, la pensée, comme exercice de la souveraineté d'un sujet qui se met en devoir de percevoir le vrai, ne peut pas être insensée » (mis en italique par l'auteur).

24. Jacques Derrida, «Cogito et histoire de la folie », p. 85 : «L'acte du Cogito vaut même si je suis fou, même si ma pensée est folle de part en part » (mis en italique par l'auteur).

25. Ibid., p. 79, note 1 : « ce qui est significatif, c'est que Descartes, au fond, ne parle jamais de la folie elle-même dans ce texte ».

26. Ce point a été souligné par Jacques Derrida, ibid., p. 90 : «Car c'est Dieu seul qui, finalement, me permettant de sortir d'un Cogito qui peut toujours rester en son moment propre une folie silencieuse, c'est Dieu seul qui garantit mes représentations et mes déterminations cognitives, c'est-à-dire mon discours contre la folie ».

27. La Possession de Loudun, présentée par Michel de Certeau, Paris, Julliard, coll. Archives, 1970.

28. Malgré les écarts sur l'interprétation de Descartes, on rejoint ici une des observations finales de Michel Foucault, Histoire de la folie, p. 70 : «Mais il s'en faut que l'histoire d'une ratio comme 
celle du monde occidental s'épuise dans le progrès d'un "rationalisme"; elle est faite, pour une part aussi grande, même si elle est plus secrète, de ce mouvement par lequel la Déraison s'est enfoncée dans notre sol, pour y disparaître, sans doute, mais y prendre racine ». Il n'est pas interdit de lire dans ce passage une réflexion sur les effets de la ratio occidentale sur le cours de l'histoire contemporaine qui mêle sous nos yeux raison et déraison jusque dans l'actualité très récente, discrètement évoquée par Alain Boureau qui voit dans la lutte des scolastiques contre les démons une guerre contre l' "Axe du Mal ", Satan hérétique, p. 141.

29. Alain Boureau, Histoires d'un historien, op. cit., p. 8.

\section{AUTEUR}

\section{ÉTIENNE ANHEIM}

École française de Rome 